\title{
Is Anterior Exenteration Necessary in Women Undergoing Radical Cystectomy?
}

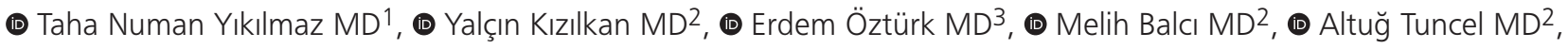 \\ (D) Cüneyt Özden MD², (1) Halil Başar MD³ \\ ${ }^{1}$ Kahramanmaraş Necip Fazıl State Hospital, Clinic of Urology, Kahramanmaraş, Turkey \\ 2 Ankara Numune Training and Research Hospital, Clinic of Urology, Ankara, Turkey \\ 3 University of Health Sciences Ankara Dr. Abdurrahman Yurtaslan Oncology Training and Research Hospital, Clinic of Urology, Ankara, Turkey
}

\begin{abstract}
Objective: Radical cystectomy is the gold standard for the treatment of muscle-invasive bladder cancer. Anterior exenteration with cystectomy is standard practice in female patients. In this study, we aimed to evaluate the necessity of anterior exenteration in female patients who underwent cystectomy for invasive bladder cancer.

Materials and Methods: Thirty-one women who underwent open radical cystectomy for bladder cancer between January 2010 and July 2018 were included in the study. The demographic characteristics, pathology results and genital organ involvement of these cases were retrospectively analyzed. Results: The mean age of the patients was 63 (range, 33-80) years. Eleven of the cases underwent anterior exenteration with cystectomy, while only two $(6 \%)$ of these cases had gynecologic organ involvement of uroepithelial origin. One patient who had positive smear sampling prior to cystectomy also had genital organ involvement in the final pathology.

Conclusion: In the light of our findings, anterior exenteration during radical cystectomy is beneficial in patients with no expectation of advanced sexual function or fertility to protect them from gynecological cancers that may develop simultaneously or later. However, in young patients and patients without suspicion of invasion in preoperative evaluation, organ sparing approaches are considered appropriate.
\end{abstract}

Keywords: Cystectomy, exenteration, bladder cancer, female, smear

\section{Introduction}

Bladder cancer is the most common urinary tract malignancy in women and is the ninth most common cancer among women in the United States (1). The risk of occurrence in men is 3-4 times higher, but women have been reported to have local advanced stage disease more frequently at the time of diagnosis (1-4). Surrounding organ involvement is frequently seen in advanced stage tumors $(3,4)$. In women, gynecologic organ involvement is reported to be very low (2.6\%) (5). Therefore, in the last decade organ sparing approaches have come up.

The main treatment principle in invasive bladder cancer is eradication of the tumor, improving the quality of life and keeping morbidity low. In anterior exenteration, uterus, fallopian tubes, ovaries and 1/3 anterior wall of vagina are excised besides bladder. With this surgery, the duration of the operation is prolonged, morbidity increases, the number of organ loss increases and postoperative sexual activity is lost. Therefore, we retrospectively evaluated the necessity of anterior exenteration in female patients who underwent cystectomy for invasive bladder cancer.

\section{Materials and Methods}

Thirty-one female patients who underwent open radical cystectomy and extended pelvic lymph node dissection for bladder cancer between January 2010 and July 2018 were eligible for the study. Demographic characteristics, follow-up times, final pathology results, genital organ involvement and operative time were recorded. Cases with and without simultaneous anterior exenteration along with radical cystectomy were divided into two groups and the variables between groups were compared. Transurethral resection (TUR) was performed with the diagnosis of primary bladder tumor before radical cystectomy in all cases and re-TUR was performed within 2-6 weeks in cases with no muscle sampling could be performed at the first resection. Cystectomy was decided because of the presence of resistant carcinoma in situ, high-grade tumor, and non-responsiveness 
to intravesical treatment in non-muscle invasive bladder tumors. Routine laboratory tests (blood biochemistry and complete blood count) and thoracoabdominopelvic computed tomography were performed in all patients for staging before surgery. Alkaline phosphatase was evaluated for possible bone metastases and cases with metastasis were not taken into surgery. Tumor-focused sections and dissected lymph nodes were stained with hematoxylin-eosin and examined under light microscope by uropathologists. The 2002 Tumor, nodes, metastases classification was used for clinical staging (6). In the pathological examination, the grading system adopted by the World Health Organization in 2004 was used (7). Ileal conduit was preferred as urinary diversion in all cases.

\section{Statistical Analysis}

Demographic and pathological data were recorded separately in cases with and without exenteration. The results were compared between the groups. Statistical analysis was performed using chi-square test through SPSS Windows 21.0 package program. $\mathrm{p}<0.05$ was accepted as the minimum limit of significance.

\section{Results}

The mean age of the 31 female patients included in the study was $63 \pm 5$ years (33-80 years), with a mean follow-up of $19 \pm 6$ months (3-41 months) (Table 1). According to the age, sociocultural level, preoperative radiological and pathological examinations, patient preference and surgeon preference, some patients underwent simultaneous anterior exenteration with radical cystectomy, while others did not. Eleven patients underwent anterior exenteration and gynecologic organ involvement was observed only in three patients (10\%), and two of these patients had bladder cancer related involvement. Tumor tissue in the rectum, vaginal cuff, cervix, endometrium, ovary and tuba was observed in one patient. The patient also had metastatic involvement in the lymph node. The case was considered as stage $\mathrm{p} 4 \mathrm{~b}$ and additional treatment was required in the postoperative period. Only one ovarian tumor was diagnosed in one of the other two cases. In the

\begin{tabular}{|c|c|}
\hline \multicolumn{2}{|l|}{ Parameters $(n=31)$} \\
\hline Mean age (years) & 63 \\
\hline Mean follow-up period, months & 19 \\
\hline \multicolumn{2}{|l|}{ Pathological stage } \\
\hline T0 & 2 \\
\hline T1 & 6 \\
\hline $\mathrm{T} 2$ & 13 \\
\hline T3 & 9 \\
\hline $\mathrm{T} 4$ & 1 \\
\hline Lymph node positivity & 4 \\
\hline Ureter surgical border involvement & 2 \\
\hline Urethral involvement & - \\
\hline Genital organ involvement & 3 \\
\hline Mean operative time, minutes & 303 \\
\hline
\end{tabular}

detailed pathological examination of the patient's sections, it was accepted that the tumor was not uroepithelial in origin, the vaginal cuff was reported as benign and the cystectomy pathology was T1G3 and the case was considered to be the second primary. The last case had cervical intraepithelial neoplasia ( $\mathrm{CIN}-2)$ pathology in smear sampling before cystectomy. Among the patients who underwent exenteration, four patients had benign gynecologic diseases including ovarian cysts in two patients and endometrial polyps in two patients, and none of the pathologies were surgical indications alone.

In two cases with gynecologic organ involvement associated with bladder cancer, the common feature was the diagnosis of high-grade muscle-invasive bladder tumors and lymph node positivity was found in both cases.

Of the 13 patients who underwent smear sampling prior to cystectomy, only one had CIN and gynecologic organ involvement was observed in the final pathology. Although smear was negative in six of 13 cases, exenteration was performed and pathology was negative. In four cases, cystectomy and exenteration were performed without smear sampling before cystectomy, and gynecologic organ involvement was observed in two of these cases.

None of the 20 patients who did not undergo simultaneous anterior exenteration with cystectomy developed invasive bladder cancer-related gynecologic organ involvement. In six of these cases, smears were taken before cystectomy and the samples were negative. There was no significant difference between the patients who underwent exenteration and who did not in terms of preoperative and postoperative complications $(p=0.2)$. There was a significant decrease in the operative time in patients who did not undergo exenteration (280 min. and 345 min., $\mathrm{p}<0.05$ ) (Table 2).

\begin{tabular}{|c|c|c|c|}
\hline & $\begin{array}{l}\text { Patients with } \\
\text { exenteration } \\
(n=11)\end{array}$ & $\begin{array}{l}\text { Patients without } \\
\text { exenteration } \\
(n=20)\end{array}$ & $\mathbf{P}$ \\
\hline Mean age (years) & 68 & 59 & 0.08 \\
\hline $\begin{array}{l}\text { Mean follow-up period, } \\
\text { months }\end{array}$ & 10 & 23 & 0.1 \\
\hline \multicolumn{4}{|l|}{ Pathological stage } \\
\hline T0 & - & 2 & - \\
\hline T1 & 2 & 4 & 0.1 \\
\hline T2 & 7 & 6 & 0.2 \\
\hline T3 & 3 & 6 & 0.2 \\
\hline T4 & 1 & - & - \\
\hline Lymph node positivity & 2 & 2 & 0.1 \\
\hline $\begin{array}{l}\text { Ureter surgical border } \\
\text { involvement }\end{array}$ & 1 & 1 & 0.1 \\
\hline Urethral involvement & - & - & - \\
\hline Genital organ involvement & 3 & - & - \\
\hline $\begin{array}{l}\text { Mean operative time, } \\
\text { minutes }\end{array}$ & 345 & 280 & $0.05^{*}$ \\
\hline $\begin{array}{l}\text { The number of cases with } \\
\text { complications }\end{array}$ & 6 & 9 & 0.2 \\
\hline
\end{tabular}


Ileal conduit method was preferred as urinary diversion in all cases. No urethral surgical margin involvement was observed in any of the cases, whereas ureteral lower end involvement was observed in two cases. Lymph node involvement was positive in four cases. Demographic and pathological evaluation of all cases is summarized in Table 1.

\section{Discussion}

In the treatment of muscle-invasive bladder cancer in women, radical cystectomy and bilateral pelvic lymph node dissection as well as anterior exenteration, which is surgical removal of uterus, upper $1 / 3$ of vagina, tuba and ovaries, is accepted as the standard approach $(8,9)$. While radical cystoprostatectomy is the gold standard treatment approach in men as $25 \%$ of prostate cancers are associated with invasive bladder cancer, anterior exenteration is still the standard practice despite the low rates of accompanying gynecological cancer in women. In studies conducted on this subject, the involvement is found to be in the range of $2.6 \%$ to $5 \%$ (5). In the study conducted by Chang et al. (9), this rate was determined as $7.5 \%$ and the authors concluded that anterior exenteration should be performed only in suspicious cases after screening gynecological organs radiologically and pathologically before surgery. In a limited number of studies on this subject, it has been reported that genital organ involvement should be investigated with radiological evaluation, smear samples and random vaginal biopsies in patients with planned gynecological organ sparing surgery before cystectomy, and anterior exenteration procedure should be performed in suspected cases $(5,8,10)$. In a study conducted in our country, genital organ involvement was observed in three (18.7\%) of 16 female cystectomy cases and anterior exenteration was added to radical cystectomy by detecting gynecologic organ involvement in two of these cases. However, in one case, vaginal cancer was found to be secondary primary, although there was a negative finding in terms of genital organ involvement in preoperative scans (8). In our study, two of the 11 patients who underwent exenteration had genital organ involvement and one had simultaneous ovarian tumor. While genital organs were radiologically examined preoperatively in all cases, vaginal smear sampling was performed additionally to 13 of them and exenteration was performed in one case diagnosed with $\mathrm{CIN}-2$. In this case, vaginal cuff involvement was observed pathologically. In the other patient with organ involvement, perivesical region and genital organ involvement were detected radiologically. Peripheral organ involvement was observed in the final pathology and it was reported as stage $4 \mathrm{~b}$. In one case, anterior exenteration was performed according to the preference of the surgeon without any suspicious condition in the preoperative scans and ovarian carcinoma was detected in this case. In cases where exenteration was not performed, no uroepithelial related genital organ tumor was detected during follow-up. When the literature is reviewed, it is reported that genital organ involvement is common in lymph node positivity, high-grade bladder tumor, bladder posterior wall involvement and bladder neck tumors and genital organ sparing should be avoided in these cases $(5,8)$. A similar presentation was observed in our results. There was no gynecologic malignancy in the follow-up period of the patients who did not have preoperative gynecologic involvement and who did not undergo anterior exenteration.

Performing anterior exenteration may lead to prolongation of operation, increased surgical morbidity and postoperative sexual dysfunction (11). Similar techniques have been developed in women, such as the prostate-sparing radical cystectomy technique in men, to protect sexual dysfunction $(12,13)$. Kolodziej et al. (13) reported a 27-year-old case who experienced pregnancy after genital organ-sparing radical cystectomy, and therefore emphasized the importance of protecting genital organs, especially in young patients considering sexual activity. Procedures such as early hormonal manipulations and oocyte freezing can be performed after surgery to prevent acute early menopause and fertility loss in patients. In our study, postoperative sexual dysfunction status could not be evaluated. However, it was found that the patients who underwent exenteration were older, and in the comparison, it was found that the operative time was significantly longer in the patients who underwent exenteration, and similarities were observed in surgical complications.

\section{Conclusion}

In conclusion, although the main principle of invasive bladder cancer surgery is eradication of tumor tissue, it is a condition that the sexual functions of the patients and therefore their quality of life should be maintained. Simultaneous anterior exenteration during radical cystectomy is beneficial in cases where there is no expectation of advanced sexual function or fertility to prevent gynecological cancers that may develop simultaneously or later. In cases where genital organ conservative radical cystectomy is decided, preoperative radiological evaluation and smear or vaginal random biopsy sampling should be performed.

\section{Ethics}

Ethics Committee Approval: Retrospective study. Informed Consent: Retrospective study.

Peer-review: Internally and externally peer-reviewed.

\section{Authorship Contributions}

Surgical and Medical Practices: T.N.Y., A.T., H.B., Concept: T.N.Y., Y.K., Design: T.N.Y., E.Ö., Data Collection or Processing: T.N.Y., Y.K., E.Ö., Analysis or Interpretation: T.N.Y., M.B., C.Ö., Literature Search: T.N.Y., Y.K., Writing: T.N.Y.

Conflict of Interest: No conflict of interest was declared by the authors.

Financial Disclosure: The authors declared that this study received no financial support.

\section{References}

1. Antoni S, Ferlay J, Soerjomataram I, et al. Bladder Cancer Incidence and Mortality: A Global Overview and Recent Trends. Eur Urol 2017;71:96-108.

2. Eser S, Zorlu F, Divrik RT, et al. Incidence and epidemiological features of cancers of the genitourinary tract in Izmir between 1993-2002. Asian Pac J Cancer Prev 2009;10:491-496.

3. Damiano R, Di Lorenzo G, Cantiello F, et al. Clinicopathologic features of prostate adenocarcinoma incidentally discovered at the 
time of radical cystectomy: An evidence-based analysis. Eur Urol 2007;52:648-657.

4. Gakis G, Schilling D, Bedke J, et al. Incidental prostate cancer at radical cystoprostatectomy: Implications for apex-sparing surgery. BJU Int 2010;105:468-471.

5. Ali-El-Dein B, Abdel-Latif M, Mosbah A, et al. Secondary malignant involvement of gynecologic organs in radical cystectomy specimens in women: is it mandatory to remove these organs routinely? J Urol 2004; 172:885-887.

6. Patriarca S, Ferretti S, Zanetti R. TNM Classification of malignant tumours - Eighth edition: which news? Epidemiol Prev 2017;41:140-143.

7. Compérat EM, Burger M, Gontero P, et al. Grading of Urothelial Carcinoma and The New "World Health Organisation Classification of Tumours of the Urinary System and Male Genital Organs 2016". Eur Urol Focus 2019;5:457-466.

8. Karadeniz T, Caner B, Topsakal M, et al. Genital organ involvement in female patients with bladder cancer: evaluation of radical cystectomy specimens. Turk J Urol 2009;35:269-273.
9. Chang SS, Cole E, Smith JA Jr, Cookson MS. Pathological findings of gynecologic organs obtained at female radical cystectomy. J Urol 2002;168:147-149.

10. Morimura Y, Nishiyama H, Hashimoto T, et al. Diagnosing endometrial carcinoma with cervical involvement by cervical cytology. Acta Cytol 2002;46:284-290.

11. Nordstrom GM, Nyman CR. Male and female sexual function and activity following ileal conduit urinary diversion. $\mathrm{Br} J$ Urol 1992;70:33-39.

12. Vallancien G, Abou El Fettouh H, Cathelineau X, et al. Cystectomy with prostate sparing for bladder cancer in 100 patients: 10-year experience. J Urol 2002;168:2413-2417.

13. Kolodziej A, Krajewski W, Tupikowski K, et al. Pregnancy and delivery in a patient with a Studer orthotopic ileal neobladder. Cent European J Urol 2016;69:431-433. 